\title{
Radiotherapy for symptom palliation of splenomegaly in patients with haematological malignancies
}

\author{
ATSUTO KATANO, AKI ISHIDA, HIDEOMI YAMASHITA and KEIICHI NAKAGAWA \\ Department of Radiology, The University of Tokyo Hospital, Bunkyo, Tokyo 113-8655, Japan
}

Received October 24, 2020; Accepted March 9, 2021

DOI: $10.3892 / \mathrm{mco} .2021 .2276$

\begin{abstract}
Splenomegaly severely compromises the quality of life of those affected. The aim of the present study was to describe the clinical characteristics of patients with haematological disorders who receive radiotherapy for splenomegaly, particularly focusing on the changes in spleen volume. The present study conducted a retrospective analysis of consecutive patients with haematological disorders who underwent splenic radiotherapy with palliative intent at the Department of Radiology of the University of Tokyo Hospital between June 2008 and June 2019. Pre- and post-radiotherapy spleen volumes were measured from computed tomography images. A total of 8 patients (5 men and 3 women) with a median age of 59 years (range, 46-76 years) were included. The median total prescription and fractional doses were 4.5 Gy (range, 1.5-10 Gy) and 0.78 Gy (range, 0.5-2.0 Gy), respectively. A total of 5 patients $(62.5 \%)$ experienced a reduction in spleen volume. The mean \pm SD spleen sizes pre- and post-radiotherapy were $1,887 \pm 1,011$ and $1,368 \pm 577 \mathrm{ml}$, respectively. The mean variation rate in spleen volume was $-19.1 \pm 24.7 \%$, and the case with the most notable improvement in the present study exhibited a $-52.4 \%$ change. Of the 5 patients who experienced pain prior to radiotherapy, 3 achieved pain relief, 1 did not experience any change and 1 patient was not assessed post-radiotherapy. Therefore, the findings of the present study revealed that palliative radiotherapy for splenomegaly may achieve symptom palliation and radiological volumetric effects in patients with haematological disorders.
\end{abstract}

\section{Introduction}

Splenomegaly is an increase in spleen size that may be caused by a number of conditions, including haematological disorders, liver diseases, infectious diseases, inflammatory diseases and primary splenic diseases (1). Regarding haematological disorders, the most common causes of splenomegaly

Correspondence to: Dr Hideomi Yamashita, Department of Radiology, The University of Tokyo Hospital, 7-3-1 Hongo, Bunkyo, Tokyo 113-8655, Japan

E-mail: yamachan07291973@yahoo.co.jp

Key words: splenomegaly, radiotherapy, palliative, volumetric effect include lymphoma, myeloproliferative neoplasms, chronic lymphocytic leukaemia and hairy cell leukaemia (2).

Symptoms associated with splenomegaly include abdominal discomfort, pain, loss of appetite and early satiety, which have a significant adverse effect on the quality of life of those affected. Moreover, when the spleen becomes hyperfunctional, a condition referred to as hypersplenism, it may lead to anaemia, immune suppression and bleeding tendency owing to the progressive destruction of blood cells (3).

Splenectomy is usually considered as the most direct intervention for splenomegaly. However, splenectomy in patients with haematological malignancies is associated with high morbidity and mortality rates owing to perioperative complications and haemostatic problems (4). In addition, patients who undergo splenectomy are at high risk of infection due to immunological changes caused by impairment of immunoglobulin synthesis, antibody-mediated clearance and phagocytic function (5). This type of infection is known as overwhelming post-splenectomy infection syndrome, which is a serious adverse event that results in a high mortality rate (6).

As a less invasive treatment, radiotherapy has been a useful treatment modality for the palliative management of patients with splenomegaly (7-9). The spleen is a highly radiosensitive organ in which radiation acts directly on extramedullary haematopoietic tissue or infiltrating lymphocytes. Splenic radiotherapy has been used for symptom relief in patients with splenomegaly or as a pre-transplantation procedure for recurrence prevention (10). Although a high response rate with symptom palliation has been reported, there are few reports on quantitative analysis of splenic irradiation. The aim of the present study was to describe the clinical characteristics of patients with haematological disorders who underwent radiotherapy for splenomegaly, particularly focusing on the radiological changes in the volume of the spleen.

\section{Materials and methods}

Patient inclusion and exclusion criteria. The present study was conducted as a retrospective analysis of existing medical records. Data were collected from consecutive patients with splenomegaly who underwent radiotherapy with palliative intent at the Department of Radiology of The University of Tokyo Hospital (Tokyo, Japan) between June 2008 and June 2019. All patients included in this study were confirmed to have haematological disorders and were not considered as feasible candidates for other treatment modalities for splenomegaly, 
such as systemic therapy or splenectomy. Patients whose clinical information after radiotherapy was not available were excluded from the study. The study protocol was approved by the Ethics Committee of our hospital and written informed consent was obtained from all patients. All adverse events were graded according to the National Cancer Institute Common Terminology Criteria for Adverse Events, version 5.0 (11).

Radiotherapy and patient evaluation. Three-dimensional conformal radiation therapy using parallel opposed or conformal beams was planned for all patients. Computed tomography (CT) scans of the patients were acquired with a slice thickness of $5.0 \mathrm{~mm}$ for planning. The entire enlarged spleen was considered as the clinical target volume (CTV). The planning target volume (PTV) was configured by considering the respiratory motions of inhalation and end-exhalation and the daily set-up margin to the CTV. The total prescription and fractional doses were set by the radiation oncologist at the time of treatment.

Following the completion of radiotherapy, the patients were examined by the physician and/or radiation oncologist, including physical examination, blood examinations and radiography. Depending on the condition of each patient, the follow-up intervals varied (range, 16-76 days). Pre- and post-radiotherapy CT images were sent to Pinnacle version. 9.10 (Philips Healthcare) for measuring the spleen volume. Pre-radiotherapy spleen volume (V1) was assessed from the CT images used in planning for radiotherapy. Post-radiotherapy spleen volume (V2) was acquired from follow-up CT images within 3 months after radiotherapy. The variation rate of spleen volume was defined as (V2-V1)/V1.

Statistical analysis. Overall survival (OS) was defined as the time from the initiation of radiotherapy until death from any cause. The OS rate curve was calculated using the Kaplan-Meier method. The difference between V1 and V2 was assessed using the Wilcoxon signed-rank test. All analyses were conducted using the $\mathrm{R}$ statistical package (The $\mathrm{R}$ Foundation for Statistical Computing). $\mathrm{P}<0.05$ was considered to indicate statistically significant differences.

\section{Results}

Patient characteristics. The characteristics of the 8 patients enrolled in the present study are listed in Table I. There were 5 men and 3 women, with a median age of 59 years (range, 46-76 years). The median total prescription and fractional doses were 4.5 Gy (range, 1.5-10 Gy) and 0.78 Gy (range, 0.5-2.0 Gy), respectively. Radiotherapy was delivered daily in 4 patients and every other day (QOD) in the remaining 4 patients. The present study included 4 patients with myelofibrosis (MF), 3 patients with acute myeloid leukaemia (including 1 patient with acute promyelocytic leukaemia) and 1 patient with chronic myeloid leukaemia. The median Karnofsky Performance Status score was 80 (range, 70-90). Patient A had been referred to the Department of Radiology at The University of Tokyo Hospital with the aim of palliation of splenomegaly before remission induction therapy. Patient $\mathrm{C}$ had developed disease progression after haematopoietic stem cell transplantation and no further chemotherapy was intended. In patient G, blast cells were still present in the bone marrow after cytosine arabinoside treatment. Patient $\mathrm{H}$ was awaiting haematopoietic stem cell transplantation after remission

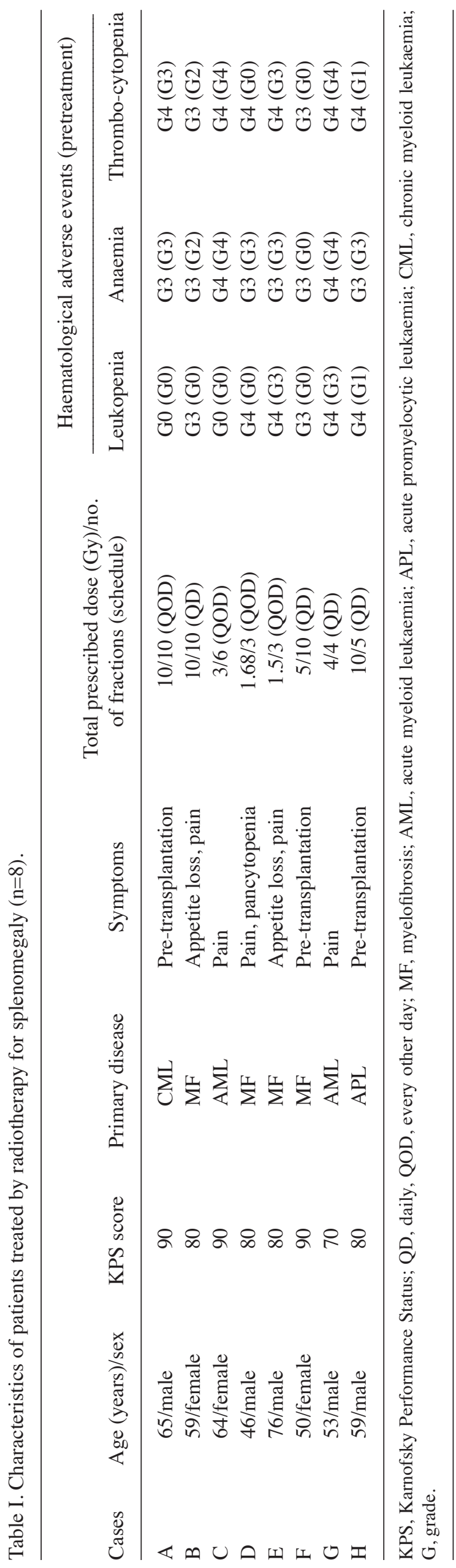




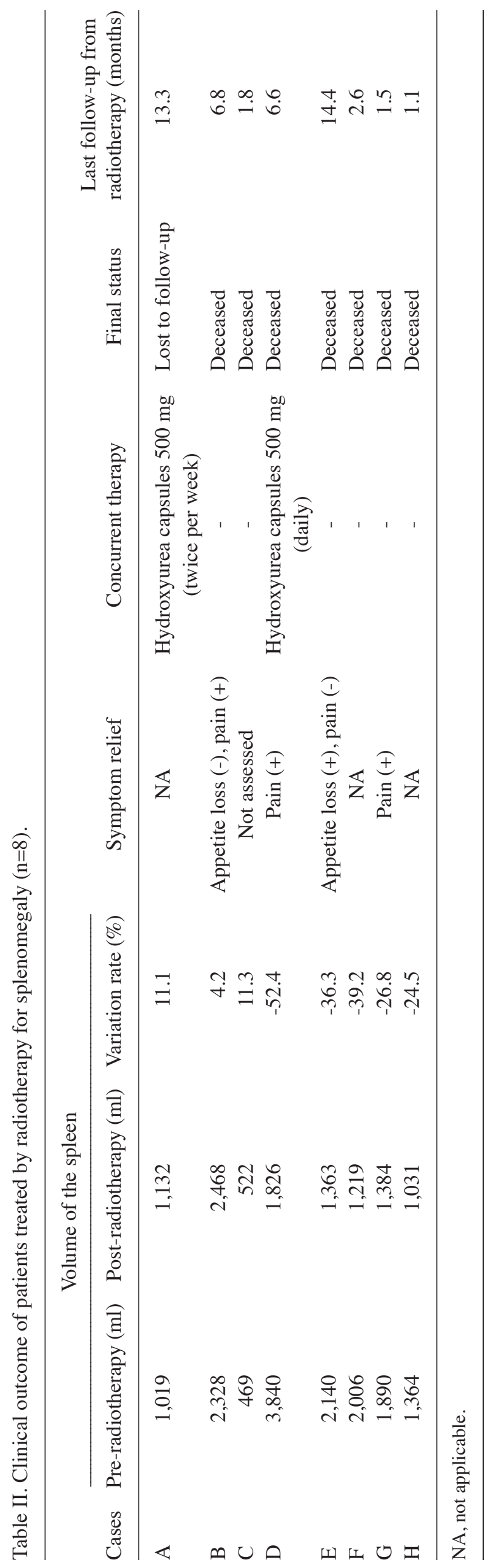

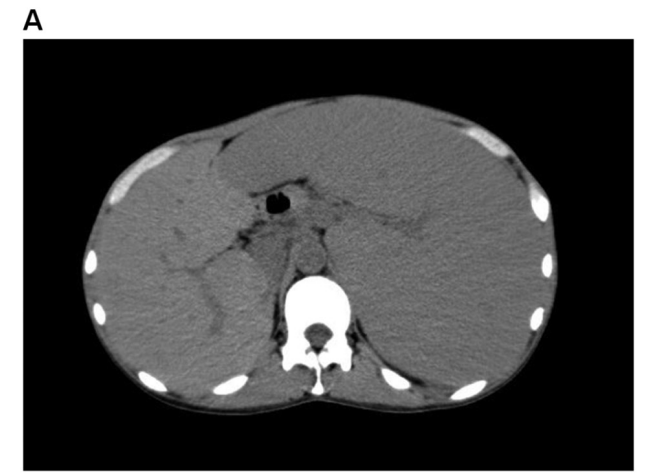

B

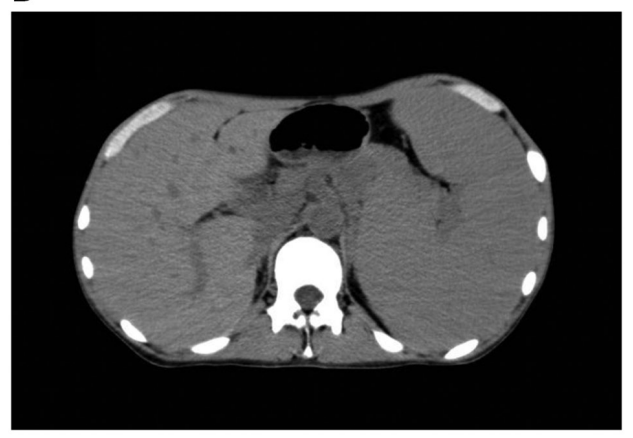

Figure 1. CT imaging was performed pre- and post-radiotherapy. (A) Pre-radiotherapy and (B) post-radiotherapy abdominal cross-sectional CT scan of patient D. CT, computed tomography.

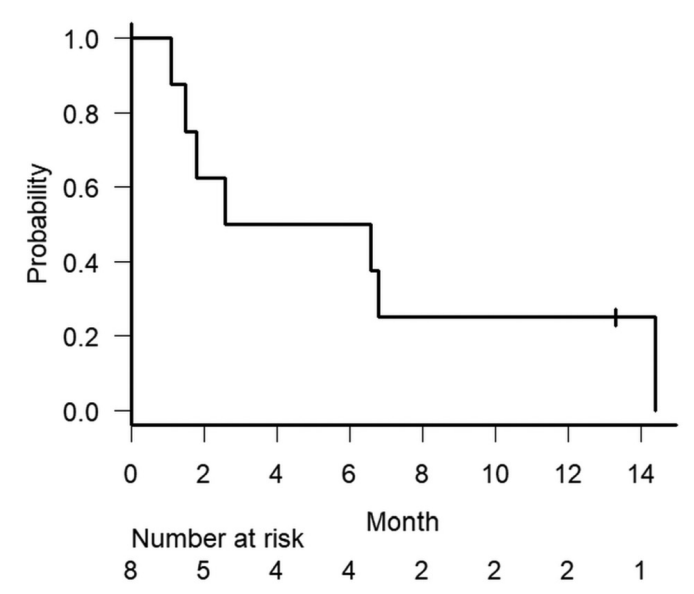

Figure 2. Kaplan-Meier survival curve for overall survival rates of the 8 patients treated by radiotherapy for splenomegaly. Vertical line, censored case.

induction therapy. Two patients were administered oral hydroxyurea capsules concurrently with radiotherapy. Splenic radiotherapy was administered to 3 patients as a pre-transplantation procedure and to 5 patients for symptom relief.

Spleen size and patient outcome. The mean \pm standard deviation pre- and post-radiotherapy spleen sizes were $1,887 \pm 1,011$ and $1,368 \pm 577 \mathrm{ml}$, respectively (Table II). The difference between the two volumes was not statistically significant $(\mathrm{P}=0.109)$, which may be attributed to the insufficient number of cases. The details of each patient after radiotherapy are presented in Table II. A total of 5 patients (62.5\%) exhibited a reduction in spleen volume. The mean variation rate in spleen volume was $-19.1 \pm 24.7 \%$. The 


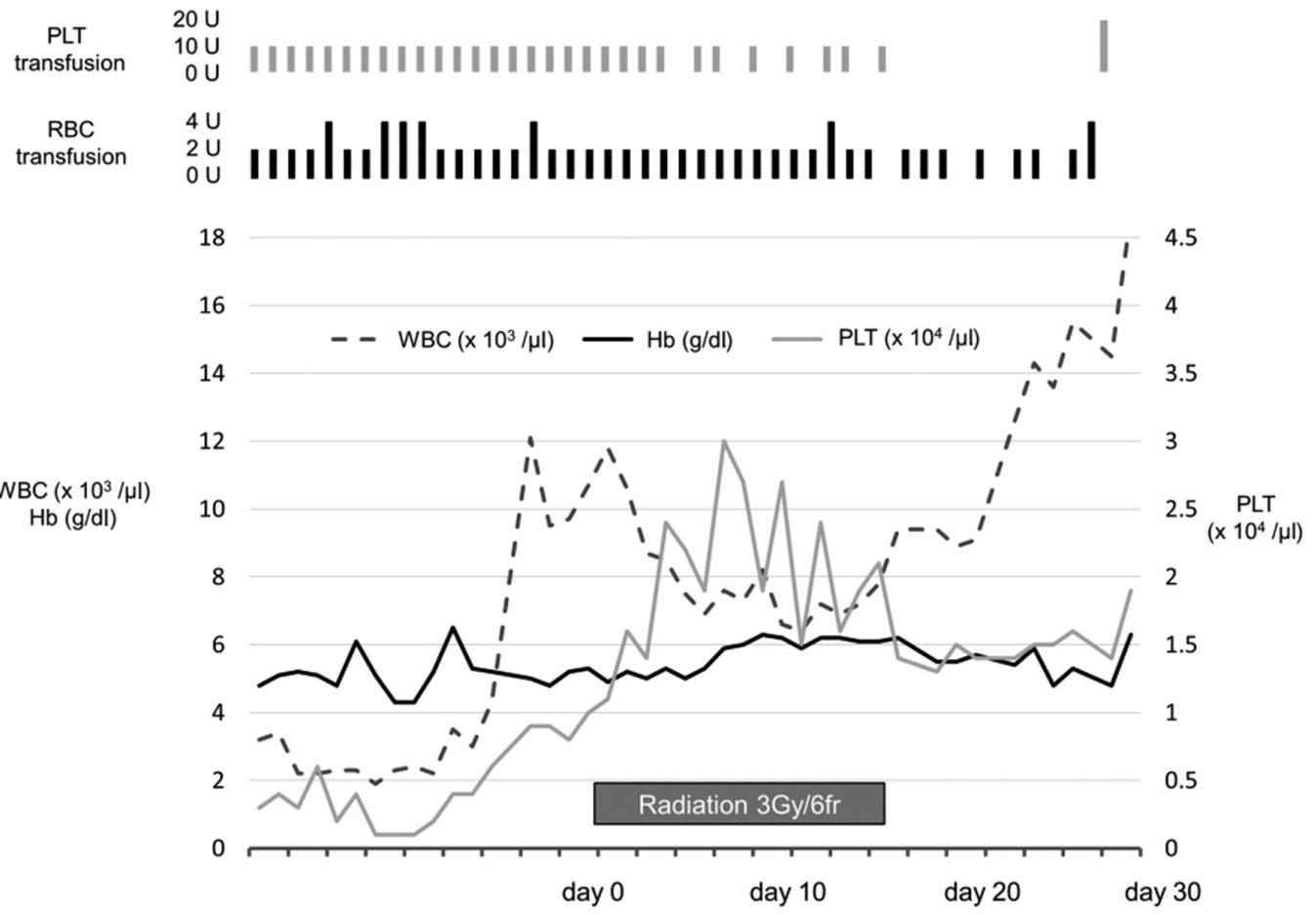

Figure 3. Time series of WBC count, Hb level and PLT count before, during and after splenic radiotherapy for patient C. Upper part of this image indicates the frequency and quantity of RBC and PLT transfusion. WBC white blood cell; Hb, haemoglobin; PLT, platelet; RBC, red blood cell.

case with the most notable improvement in the present study was patient $\mathrm{D}$, who exhibited a-52.4\% reduction in spleen size compared with pre-radiotherapy (Fig. 1). Of the 5 patients who experienced pain prior to radiotherapy, 3 patients achieved pain relief, 1 patient did not experience any change and 1 patient was not assessed after radiotherapy. Of the 2 patients who reported loss of appetite before radiotherapy, 1 patient achieved symptom relief and 1 patient did not experience any change.

At the time of analysis, 7 patients had died owing to exacerbation of the primary disease and 1 patient was lost to follow-up. The median survival time and 1-year OS rate were 4.6 months [95\% confidence interval (CI): 1.1-upper limit not available] and 25.0\% (95\% CI: 3.7-55.8), respectively (Fig. 2). At 3 months from the beginning of radiotherapy, 4 patients remained alive. Although splenic volume reduction was not achieved in patient $\mathrm{A}$, haematopoietic stem cell transplantation was performed as planned. Patient B suffered from exacerbation of splenomegaly due to primary disease progression. In patients $\mathrm{D}$ and $\mathrm{E}$, the volume of the spleen had been reduced and remained stable.

Adverse events. Although severe (grade 3 or 4) haematological adverse events were observed in almost all patients, the haematological condition prior to radiotherapy was very poor (Table I) owing to the primary disease and hypersplenism due to splenomegaly. Hypersplenism causes pancytopenia as a result of the excessive destruction of premature blood cells. Splenic irradiation is considered to be effective for palliation of hypersplenism. Patient C, a 64-year-old female with acute myeloid leukaemia, required daily red blood cell and platelet transfusion. The patient was referred to the Department of Radiology with the aim of palliation for abdominal pain and pancytopenia due to hypersplenism. During radiotherapy with
0.5 Gy per fraction QOD to a total dose of $3 \mathrm{~Gy}$, the platelet count gradually improved; in addition, after radiotherapy, the frequency of transfusions decreased, as shown in Fig. 3. No significant late radiation-induced adverse events were observed.

\section{Discussion}

There is currently no clear consensus on palliative radiotherapy for splenomegaly in lymphatic and myeloid malignancies. However, this method has been reported in several retrospective studies or case series. Kriz et al (12) reported their retrospective cohort analysis of 246 courses in 122 patients with haematological disorders who underwent radiotherapy for splenomegaly. They achieved an improvement of $74.8 \%$ in pain and $73.6 \%$ in blood parameters of the patients who received splenic radiotherapy, which comprised a total prescription dose of 0.3-16 Gy and fractional dose of 0.1-2 Gy. In addition, a systematic review of 766 irradiation courses in 486 patients reported that the most commonly used total dose and fraction scheme was 10 Gy in 10 fractions over 2 weeks (13). The review reported objective response rates for palliation of splenomegaly symptoms in $85-90 \%$ of the patients. Moreover, they reported no statistically significant correlation between dose and symptom relief, indicating that lower doses may be sufficient for symptom palliation. In a recent study, low-dose QOD radiotherapy with an initial total prescribed dose of $10 \mathrm{~Gy}$ and fractional dose of $0.5 \mathrm{~Gy}$ was used, and the median reduction value of the superior-to-inferior spleen size was $4.6 \mathrm{~cm}$ (14).

Splenic irradiation is also effective in cases of congestive splenomegaly. Bruns et al (15) reported that low-dose splenic irradiation was effective in a retrospective analysis of 5 patients with liver disease. In their report, all patients who 
experienced abdominal pain prior to irradiation achieved pain relief. In the 2 patients whose spleen size could be measured after radiotherapy, there was $\sim 10 \%$ reduction in the spleen size. Liu et al (16) also reported 5 cases of patients with congestive splenomegaly and a history of liver cirrhosis. They used daily radiotherapy with a total prescription dose of 12 Gy in 8 fractions. All patients in that study experienced palliation of thrombocytopenia due to splenic irradiation. In addition, the spleen size decreased by 15 and $70 \%$ in 2 patients, whereas no remarkable response was observed in the remaining 3 patients.

The precise mechanisms of action of splenic radiotherapy remain unclear. Although radiotherapy exerts direct effects on haematopoietic and lymphoid cells in the spleen, the immunological response is also considered to play an important role. Radiation-induced inflammatory cytokines, such as IL-2 and TNF- $\alpha$, contribute to the systemic response to splenic irradiation (17). Moreover, the differences in lymphocyte subsets with respect to sensitivity to radiation cause proportional changes in lymphocyte subsets in the spleen after radiation; these proportional changes cause immune system modulation in the patients (18).

The present study included 4 patients with MF. Patients with MF tend to have a higher frequency of splenomegaly at the time of diagnosis, which is a poor prognostic factor (19). For patients with MF, Janus kinase (JAK) inhibitors have demonstrated an effective decrease in splenomegaly (20). An oral JAK1/JAK2 inhibitor, ruxolitinib, has been shown to reduce splenomegaly, improve symptoms and improve survival and prognosis as demonstrated in the phase 3 COMFORT studies $(21,22)$. A selective oral JAK2 inhibitor, fedratinib, which was recently approved by the US Food and Drug Administration, was also found to be effective in reducing spleen volume (23). Moreover, newer generation JAK inhibitors, momelotinib and pacritinib, are being evaluated in phase 3 trials and may be considered as a treatment option (24).

There were certain limitations to the present study. The major limitation was the small sample size, due to which statistically significant conclusions could not be established. Additionally, the heterogeneity of patient characteristics and treatment modalities included in the present study also represents a limitation. The radiotherapy in the present study was heterogeneous with respect to both the total prescription and fractional doses. Moreover, the heterogeneity of the treatment assessment is also considered as a limitation owing to the retrospective nature of the analysis.

In conclusion, the present study revealed that palliative radiotherapy for splenomegaly in patients with haematological disorders may be effective in achieving symptom relief as well as in terms of the volumetric effect. However, the optimal total dose and fractionation schedule of palliative radiotherapy for splenomegaly requires further investigation and additional evidence before reaching definitive conclusions.

\section{Acknowledgements}

Not applicable.

\section{Funding}

No funding was received.

\section{Availability of materials and data}

All datasets generated or analysed during this study are included in this published article.

\section{Authors' contributions}

AK wrote the original draft and conducted statistical analyses. AK and AI collected and interpreted the clinical data. HY conceived the present study and participated in the study design and coordination. KN made substantial contributions to the interpretation of the data. All authors have revised this report critically for important intellectual content. AK and HY confirm the authenticity of all the raw data. All the authors have read and approved the final manuscript.

\section{Ethics approval and consent to participate}

The study protocol was approved by the Ethics Committee of our hospital and written informed consent was obtained from all patients.

\section{Patient consent for publication}

Not applicable.

\section{Competing interests}

The authors declare that they have no competing interests.

\section{References}

1. Pozo AL, Godfrey EM and Bowles KM: Splenomegaly: Investigation, diagnosis and management. Blood Rev 23: 105-111, 2009.

2. Curovic Rotbain E, Lund Hansen D, Schaffalitzky de Muckadell O, Wibrand F, Meldgaard Lund A and Frederiksen H: Splenomegaly-diagnostic validity, work-up, and underlying causes. PLoS One 12: e0186674, 2017.

3. Lv Y, Lau WY, Li Y, Deng J, Han X, Gong X, Liu N and Wu H: Hypersplenism: History and current status. Exp Ther Med 12: 2377-2382, 2016.

4. Bagrodia N, Button AM, Spanheimer PM, Belding-Schmitt ME, Rosenstein LJ and Mezhir JJ: Morbidity and mortality following elective splenectomy for benign and malignant hematologic conditions: Analysis of the American college of surgeons national surgical quality improvement program data. JAMA Surg 149: 1022-1029, 2014.

5. Luu S, Spelman D and Woolley IJ: Post-splenectomy sepsis: Preventative strategies, challenges, and solutions. Infect Drug Resist 12: 2839-2851, 2019.

6. Sinwar PD: Overwhelming post splenectomy infection syndrome-review study. Int J Surg 12: 1314-1316, 2014.

7. Paulino AC and Reddy SP: Splenic irradiation in the palliation of patients with lymphoproliferative and myeloproliferative disorders. Am J Hosp Palliat Care 13: 32-35, 1996.

8. McFarland JT, Kuzma C, Millard FE and Johnstone PA: Palliative irradiation of the spleen. Am J Clin Oncol 26: 178-183, 2003.

9. Soldić Z, Murgić J, Jazvić M, Radić J, Bolanca A, Stancić V and Kusić Z: Splenic irradiation in hematologic malignancies and other hematologic disorders-single institution experience. Acta Clin Croat 50: 29-35, 2011.

10. Gratwohl A, Hermans J, van Biezen A, Arcese W, Debusscher L, Ernst P, Ferrant A, Frassoni F, Gahrton G, Iriondo A, et al: Splenic irradiation before bone marrow transplantation for chronic myeloid leukaemia. Chronic leukaemia working party of the European group for blood and marrow transplantation (EBMT). Br J Haematol 95: 494-500, 1996. 
11. Common Terminology Criteria for Adverse Events (CTCAE), version 5.0. Washington, DC: US Department of Health and Human Services. https://ctep.cancer.gov/protocolDevelopment/electronic_applications/docs/CTCAE_v5_Quick_Reference_5x7.pdf. Accessed February 28, 2021.

12. Kriz J, Micke O, Bruns F, Haverkamp U, Mücke R, Schäfer U, Seegenschmiedt H, Müller RP and Eich HT: Radiotherapy of splenomegaly: A palliative treatment option for a benign phenomenon in malignant diseases. Strahlenther Onkol 187: 221-224, 2011.

13. Zaorsky NG, Williams GR, Barta SK, Esnaola NF, Kropf PL, Hayes SB and Meyer JE: Splenic irradiation for splenomegaly: A systematic review. Cancer Treat Rev 53: 47-52, 2017.

14. de la Pinta C, Fernández Lizarbe E, Montero Luis Á, Domínguez Rullán JA and Sancho García S: Treatment of symptomatic splenomegaly with low doses of radiotherapy: Retrospective analysis and review of the literature. Tech Innov Patient Support Radiat Oncol 3-4: 23-29, 2017.

15. Bruns F, Bremer M, Dettmer A and Janssen S: Low-dose splenic irradiation in symptomatic congestive splenomegaly: Report of five cases with literature data. Radiat Oncol 9: 86, 2014.

16. Liu MT, Hsieh CY, Chang TH, Lin JP and Huang CC: Radiotherapy for hypersplenism from congestive splenomegaly. Ann Saudi Med 24: 198-200, 2004.

17. Paulsen $\varnothing$, Laird B, Aass N, Lea T, Fayers P, Kaasa S and Klepstad P: The relationship between pro-inflammatory cytokines and pain, appetite and fatigue in patients with advanced cancer. PLoS One 12: e0177620, 2017.
18. Weinmann M, Becker G, Einsele H and Bamberg M: Clinical indications and biological mechanisms of splenic irradiation in chronic leukaemias and myeloproliferative disorders. Radiother Oncol 58: 235-246, 2001.

19. Gangat N, Caramazza D, Vaidya R, George G, Begna K, Schwager S, Van Dyke D, Hanson C, Wu W, Pardanani A, et al: DIPSS plus: A refined dynamic international prognostic scoring system for primary myelofibrosis that incorporates prognostic information from karyotype, platelet count, and transfusion status. J Clin Oncol 29: 392-397, 2011.

20. Tremblay D, Schwartz M, Bakst R, Patel R, Schiano T, Kremyanskaya M, Hoffman R and Mascarenhas J: Modern management of splenomegaly in patients with myelofibrosis. Ann Hematol 99: 1441-1451, 2020.

21. Verstovsek S, Mesa RA, Gotlib J, Levy RS, Gupta V, DiPersio JF, Catalano JV, Deininger M, Miller C, Silver RT, et al: A double-blind, placebo-controlled trial of ruxolitinib for myelofibrosis. N Engl J Med 366: 799-807, 2012.

22. Harrison C, Kiladjian JJ, Al-Ali HK, Gisslinger H, Waltzman R, Stalbovskaya V, McQuitty M, Hunter DS, Levy R, Knoops L, et al: JAK inhibition with ruxolitinib versus best available therapy for myelofibrosis. N Engl J Med 366: 787-798, 2012.

23. Talpaz M and Kiladjian JJ: Fedratinib, a newly approved treatment for patients with myeloproliferative neoplasm-associated myelofibrosis. Leukemia 35: 1-17, 2021.

24. Patel AA and Odenike O: The next generation of JAK inhibitors: An update on fedratinib, momelotonib, and pacritinib. Curr Hematol Malig Rep 15: 409-418, 2020. 